\title{
Comparing the Characteristics and Predicting the Survival of Head and Neck Melanoma versus Body Melanoma: A Population-based Study
}

\section{Yuxin Ding}

Department of Dermatology, The Second Affiliated Hospital, School of Medicine, Zhejiang University, Hangzhou, China, No. 88, Jiefang Road, Hangzhou 310009

\section{Runyi Jiang}

Spinal Tumor Center, Department of Orthopaedic Oncology, Changzheng Hospital, Second Military Medical University, Shanghai, 200003

\section{Yuhong Chen}

Department of Dermatology, The Second Affiliated Hospital, School of Medicine, Zhejiang University, Hangzhou, China, No. 88, Jiefang Road, Hangzhou 310009

\section{Jing Jing}

Department of Dermatology, The Second Affiliated Hospital, School of Medicine, Zhejiang University, Hangzhou, China, No. 88, Jiefang Road, Hangzhou 310009

\section{Xiaoshuang Yang}

Department of Dermatology, The Second Affiliated Hospital, School of Medicine, Zhejiang University, Hangzhou, China, No. 88, Jiefang Road, Hangzhou 310009

\section{Xianjie Wu}

Department of Dermatology, The Second Affiliated Hospital, School of Medicine, Zhejiang University, Hangzhou, China, No. 88, Jiefang Road, Hangzhou 310009

\section{Xiaoyang Zhang}

Department of Dermatology, The Second Affiliated Hospital, School of Medicine, Zhejiang University, Hangzhou, China, No. 88, Jiefang Road, Hangzhou 310009

\section{Jiali Xu}

Department of Dermatology, The Second Affiliated Hospital, School of Medicine, Zhejiang University, Hangzhou, China, No. 88, Jiefang Road, Hangzhou 310009

\section{Piaopiao Xu}

Department of Dermatology, The Second Affiliated Hospital, School of Medicine, Zhejiang University, Hangzhou, China, No. 88, Jiefang Road, Hangzhou 310009

\section{ShuChen LiuHuang}

China Academy of Art, Hangzhou, 310000

\section{Zhongfa Lu ( $\sim$ lzfskin@zju.edu.cn )}

Department of Dermatology, The Second Affiliated Hospital, School of Medicine, Zhejiang University, Hangzhou, China, No. 88, Jiefang Road, Hangzhou 310009 


\section{Research Article}

Keywords: head and neck melanoma, nomogram, prognostic factors, survival, SEER

Posted Date: February 16th, 2021

DOl: https://doi.org/10.21203/rs.3.rs-154802/v2

License: (c) (1) This work is licensed under a Creative Commons Attribution 4.0 International License. Read Full License

Version of Record: A version of this preprint was published at BMC Cancer on April 16th, 2021. See the published version at https://doi.org/10.1186/s12885-021-08105-y. 


\section{Abstract}

Background: Previous studies have reported poorer survival in head and neck melanoma (HNM) than in body melanoma (BM). Individualized tools to predict the prognosis for patients with HNM or BM remain insufficient. We aim to compare the characteristics of HNM and BM, and establish and validate the nomograms for predicting the 3-, 5- and 10-year survival of patients with HNM or BM.

Methods: We studied patients with HNM or BM from 2004 to 2015 in the Surveillance, Epidemiology, and End Results (SEER) database. The HNM group and BM group were randomly divided into training and validation cohorts. We performed the Kaplan-Meier method for survival analysis, and used multivariate Cox proportional hazards models to identify independent prognostic factors. Nomograms for HNM patients or BM patients were developed via the rms package, and were measured by the concordance index (C-index), the area under the receiver operator characteristic (ROC) curve (AUC) and calibration plots.

Results: Of 70605 patients acquired, $21 \%(n=15071)$ had HNM and $79 \%(n=55534)$ had BM. The HNM group contained more older patients, male patients, and lentigo maligna melanoma, and more frequently had thicker tumors and metastases than the BM group. The 5 -year CSS and OS rates were $88.1 \pm 0.3 \%$ and $74.4 \pm 0.4 \%$ in the HNM group and $92.5 \pm 0.1 \%$ and $85.8 \pm 0.2 \%$ in the BM group, respectively. Eight independent prognostic factors (age, sex, histology, thickness, ulceration, stage, metastases, and surgery) were identified to construct nomograms for HNM patients or BM patients. The performance of the nomograms were excellent: the C-index of the CSS prediction for HNM patients and BM patients in the training cohort were 0.839 and 0.895 , respectively; in the validation cohort, they were 0.848 and 0.888 , respectively; the AUCs for the 3-, 5- and 10-year CSS rates of HNM were $0.871,0.865$ and 0.854 (training), and 0.881, 0.879 and 0.861 (validation), respectively; of BM, the AUCs were $0.924,0.918$ and 0.901 (training) and $0.916,0.908$ and 0.893 (validation), respectively; and the calibration plots showed great consistency.

Conclusions: The characteristics of HNM and BM are heterogeneous, and we constructed and validated specific nomograms as practical prognostic tools for patients with HNM or BM.

\section{Background}

Worldwide, cutaneous melanoma is an important public health problem and accounts for $1.7 \%$ of all newly diagnosed primary malignant tumors annually[1]. In the United States, the incidence of cutaneous melanoma continues to rise, and the 5-year relative survival rate is $92 \%$ (2009-2015)[2]. Many studies have reported that anatomic location is an important prognostic factor for primary cutaneous melanoma[3-5], and head and neck melanoma (HNM) should be treated differently from melanoma in other regions (body melanoma, BM) [6-9]. The head and neck areas are more likely to be exposed to chronic and continued sun exposure, while other body areas usually receive intermittent UV radiation[10,11]. When considering the cancer density, cutaneous melanoma occurs more frequently in the head and neck regions than in the trunk and extremity regions[7-9]. In addition, many studies have reported poorer survival in HNM[12-14]. Therefore, a further understanding of the characteristics and prognostic factors of HNM and BM is necessary to help clinical management. 
To date, limited studies have compared the clinical features of HNM and BM patients based on a large population $[9,14,15]$, and few have compared the prognostic factors of the two subsites[16]. Although several studies estimated that some variables, such as age, sex, histology and thickness, were independent prognostic factors for $\operatorname{HNM}[15,17,18]$, no integrated tool that utilizes all available information to comprehensively predict the survival rate of HNM patients has been developed. A nomogram is a widely used graphical depiction of a predictive tool that can provide the overall probability of a specific outcome for any patient $[19,20]$. Nomograms can integrate all sorts of factors to estimate tumor prognosis and have been confirmed to be a reliable instrument in numerous survival analysis studies[21, 22]. Consequently, we performed a population-based study to compare the clinicopathological characteristics and treatments of HNM and BM and to identify independent prognostic factors of the two subgroups. We aimed to establish and validate the nomograms for predicting the 3-, 5- and 10-year survival of patients with HNM or BM.

\section{Methods}

\subsection{Patient selection}

The information of eligible patients from 2004 to 2015 was obtained using SEER*Stat (version 8.3.8) from the Surveillance, Epidemiology, and End Results (SEER) database. In general, extracting SEER data does not require informed patient consent, and the SEER database does not provide case-identifying information. As a consequence, approval from the institutional review board is not required.

In this study, the inclusion criteria were as follows: 1) patients had a confirmed diagnosis of malignant cutaneous melanoma with ICD-0-3/WHO 2008 morphology codes 8721-8723, 8726-8728, 8730, 8740$8746,8760-8761,8770-8774,8780$ and $8790 ; 2)$ the codes of the primary site were $C 440-C 447 ; 3)$ patients acquired a diagnosis with a living status; and 4) patients had active follow-up. The exclusion criteria were as follows: 1) clinical diagnosis without pathological reports; 2) acquired diagnosis from autopsy or death certificate only; and 3) unknown survival months. Fifteen variables were extracted from the SEER program, including age, sex, race, histology, anatomic site, thickness, ulceration, stage, metastases, primary site surgery, radiation, chemotherapy, survival time, overall survival (OS) status and cancer-specific survival (CSS) status. We excluded patients who did not have complete information on all the above variables. The stage in the SEER program referred to the stage of lymph nodes, including localized, regional and distant lymph nodes.

CSS was defined as the duration from the first day of follow-up to the day of death due to malignant cutaneous melanoma or the day of the last follow-up, while OS was defined as the time from the first day of follow-up to death from any cause or the end of the follow-up. Patients whose OS was recorded as dead but had 0 survival months in SEER were assigned a survival time of 0.5 months for analysis, while patients alive at follow-up with 0 survival months were excluded. Patients with anatomic site codes C440- Skin of lip, C441Eyelid, C442- External ear, C443- Skin other/unspecific parts of face, or C444- Skin of scalp and neck were assigned to the HNM group, while those whose site was coded as C445- Skin of trunk, C446- Skin of upper limb and shoulder, or C447- Skin of lower limb and hip were assigned to the BM group. 
Ultimately, a total of 70605 malignant cutaneous melanoma patients (15071 HNM patients and 55534 BM patients) were acquired for further analysis. The patient selection workflow is shown in Fig. 1.

\subsection{Statistical analysis}

Qualitative data are described as numbers and percentages, and quantitative data are described as means with standard deviations (SDs) and ranges. X-tile software (Yale University, New Haven, CT, USA) was employed to define the optimal cutoff values of continuous variables. X-tile software divided age into three subgroups for all possible divisions and selected the optimal cutoff value based on the highest chi-square value calculated by Kaplan-Meier survival analysis and the log-rank test. According to CSS, the optimal cutoff values for age were subdivided into $<=58,59-78$ and $>=79$ years, while based on OS, the optimal cutoff points were $<=63,64-79$, and $>=80$ years (Fig. 1). Melanoma thickness was classified into five subsets based on the American Joint Committee on Cancer (AJCC) staging system and the thin melanoma study (< 0.6 mm, 0.6-1.0 mm, 1.01-2.0 mm, 2.01-4.0 mm, and > 4.0 mm)[23].

For nomogram construction and validation, the HNM group and BM group were randomly divided into training and validation cohorts (split ratio: 7:3). Chi-square analysis or Fisher's exact test was performed to compare the baseline characteristics between the HNM group and BM group and to calculate the associations between the training cohort and the validation cohort. $\mathrm{P}<0.05$ was considered significant.

In the univariate analysis, the Kaplan-Meier method and the log-rank test were conducted to determine significant prognostic factors in the training cohort for CSS or OS by SPSS 25.0 (SPSS Inc., Chicago, IL, USA). Variables with $\mathrm{p}<0.1$ were included in the multivariate Cox proportional hazards model to determine the independent prognostic factors $(p<0.05)$. Furthermore, corresponding hazard ratios were calculated. GraphPad Prism 8.0 (GraphPad Software, San Diego, CA, USA) was used to create survival analysis curves for the independent prognostic factors. Notably, as specific information for radiotherapy and chemotherapy was not provided in the SEER database, we did not conduct survival analysis for the two factors.

\subsection{Nomogram construction and validation}

In the HNM group and BM group, nomograms based on the results of the Cox regression model were developed for the 3-year, 5-year and 10-year CSS rates via the rms package in R (version 4.0.1). Predictive prognostic nomograms for OS were also constructed for the two groups. The performance and predictive value of the nomograms were measured by the concordance index (C-index) and the area under the receiver operator characteristic (ROC) curve (AUC). In addition, calibration plots were used to assess the prediction accuracy of the nomograms by comparing the predicted values with the actual outcomes.

\section{Results}

\subsection{Comparing the characteristics of head and neck melanoma versus body melanoma}


A total of 70605 eligible patients diagnosed with malignant cutaneous melanoma from 2004 to 2015 were evaluated in this study, and the total population was divided into an HNM group and a BM group according to the anatomic site of the tumor.

The comparison of the two groups are summarized in table 1. The HNM cohort contained 15071 (21\%) patients, and the BM cohort contained 55534 (79\%) patients. Compared with the BM group, the HNM group contained more older patients (mean age 64.6 vs. 57.2 years) and male patients (72.4\% vs. 52.3\%).

Superficial spreading melanoma (SSM) was the most common histological type in both groups, but lentigo maligna melanoma (LMM) was much more frequent in the HNM group than in the BM group (31.8\% vs. $6.6 \%)$. HNM patients were diagnosed at a thicker stage, more frequently had metastases and were more often treated with radiation than BM patients. The mean survival time was 60.59 months in the HNM group and 67.76 months in the BM group. The 5-year CSS and OS rates were $88.1 \pm 0.3 \%$ and $74.4 \pm 0.4 \%$ in the HNM group and $92.5 \pm 0.1 \%$ and $85.8 \pm 0.2 \%$ in the BM group, respectively. In addition, the Kaplan-Meier curves showed that the prognosis of patients with BM was better than that of patients with HNM (Fig. 2).

$\mathrm{HNM}=$ head and neck melanoma; $\mathrm{BM}=$ body melanoma.

All HNM patients were randomly divided into training $(n=10551)$ and validation $(n=4520)$ cohorts. Using the same method, $38876 \mathrm{BM}$ patients were assigned to the training cohort, and $16658 \mathrm{BM}$ patients were assigned to the validation cohort. Tables 2 shows that there were no significant differences between the training and validation cohorts. 
Table 2

The characteristics of patients between the training cohort and the validation cohort

\begin{tabular}{|c|c|c|c|c|c|c|}
\hline & \multicolumn{3}{|c|}{ the HNM group } & \multicolumn{3}{|l|}{ the BM group } \\
\hline & $\begin{array}{l}\text { Training } \\
\text { cohort (\%) }\end{array}$ & $\begin{array}{l}\text { Validation } \\
\text { cohort (\%) }\end{array}$ & $\begin{array}{l}P \\
\text { value }\end{array}$ & $\begin{array}{l}\text { Training } \\
\text { cohort (\%) }\end{array}$ & $\begin{array}{l}\text { Validation } \\
\text { cohort (\%) }\end{array}$ & $\begin{array}{l}P \\
\text { value }\end{array}$ \\
\hline Total & 10551(70.0) & $4520(30.0)$ & & $38876(70.0)$ & 16658(30.0) & \\
\hline Age & & & 0.21 & & & 0.748 \\
\hline$<=58$ & 3349 (31.7) & 1467 (32.5) & & $20275(52.2)$ & $8666(52.0)$ & \\
\hline $59-78$ & 4885 (46.3) & $2023(44.8)$ & & $14790(38.0)$ & $6324(38.0)$ & \\
\hline >=79 & 2317 (22.0) & $1030(22.8)$ & & 3811 (9.8) & 1668 (10.0) & \\
\hline Sex & & & 0.62 & & & 0.836 \\
\hline Male & 7653 (72.5) & 3260 (72.1) & & $20359(52.4)$ & 8707 (52.3) & \\
\hline Female & 2898 (27.5) & $1260(27.9)$ & & $18517(47.6)$ & 7951 (47.7) & \\
\hline Race & & & 0.911 & & & 0.31 \\
\hline White & 10452 (99.1) & 4476 (99.0) & & 38267 (98.4) & $16417(98.6)$ & \\
\hline a Others & $99(0.9)$ & $44(1.0)$ & & 609 (1.6) & $241(1.4)$ & \\
\hline Histology & & & 0.31 & & & 0.067 \\
\hline NM & $1680(15.9)$ & $672(14.9)$ & & $5448(14.0)$ & 2457 (14.7) & \\
\hline LMM & 3328 (31.5) & 1470 (32.5) & & $2589(6.7)$ & $1049(6.3)$ & \\
\hline SMM & $4306(40.8)$ & 1862 (41.2) & & $27177(69.9)$ & $11571(69.5)$ & \\
\hline $\begin{array}{l}\text { b Other } \\
\text { Melanoma }\end{array}$ & 1237 (11.7) & $516(11.4)$ & & 3662 (9.4) & $1581(9.5)$ & \\
\hline Thickness & & & 0.38 & & & 0.013 \\
\hline$<0.6 \mathrm{~mm}$ & 4691 (44.5) & $2026(44.8)$ & & $17866(46.0)$ & 7435 (44.6) & \\
\hline $0.6-1.0 \mathrm{~mm}$ & 1814 (17.2) & 787 (17.4) & & 8410 (21.6) & 3588 (21.5) & \\
\hline $1.01-2.0 \mathrm{~mm}$ & $1666(15.8)$ & $728(16.1)$ & & $6073(15.6)$ & $2673(16.0)$ & \\
\hline $2.01-4.0 \mathrm{~mm}$ & 1268 (12.0) & 491 (10.9) & & 3666 (9.4) & $1671(10.0)$ & \\
\hline$>4.0 \mathrm{~mm}$ & $1112(10.5)$ & $488(10.8)$ & & $2861(7.4)$ & $1291(7.8)$ & \\
\hline Ulceration & & & 0.459 & & & 0.43 \\
\hline No & 8841 (83.8) & 3810 (84.3) & & $33040(85.0)$ & $14113(84.7)$ & \\
\hline Yes & 1710 (16.2) & 710 (15.7) & & $5836(15.0)$ & 2545 (15.3) & \\
\hline
\end{tabular}




\begin{tabular}{|c|c|c|c|c|c|c|}
\hline & the HNM grou & & & the BM group & & \\
\hline Stage & & & 0.795 & & & 0.159 \\
\hline Localized & 9051 (85.8) & $3893(86.1)$ & & 34591 (89.0) & $14729(88.4)$ & \\
\hline Regional & $1320(12.5)$ & $548(12.1)$ & & $3731(9.6)$ & 1677 (10.1) & \\
\hline Distant & $180(1.7)$ & $79(1.7)$ & & $554(1.4)$ & $252(1.5)$ & \\
\hline Metastases & & & 0.937 & & & 0.251 \\
\hline No & 10405 (98.6) & 4456 (98.6) & & 38501 (99.0) & $16479(98.9)$ & \\
\hline Yes & $146(1.4)$ & $64(1.4)$ & & $375(1.0)$ & $179(1.1)$ & \\
\hline Surgery & & & 0.545 & & & 0.591 \\
\hline No & 245 (2.3) & $97(2.1)$ & & $773(2.0)$ & 319 (1.9) & \\
\hline Yes & 10306 (97.7) & 4423 (97.9) & & $38103(98.0)$ & $16339(98.1)$ & \\
\hline
\end{tabular}

\subsection{Identification of prognostic factors}

The univariate analysis revealed that nine variables (age, sex, race, histology, thickness, ulceration, stage, metastases, and surgery) were potential clinical determinants of CSS in the HNM group. In addition, the same nine significant factors were identified in the BM group. Table 3 shows the 5-year CSS and OS rates for the subgroups. The factors above were further identified by multivariate Cox proportional hazards models, in which the results revealed that eight variables (age, sex, histology, thickness, ulceration, stage, metastases, and surgery) were independent prognostic factors in the HNM training cohort, and the same eight independent prognostic factors were identified in the BM training cohort (Table 4). The survival analysis curves for the identified independent factors of CSS for the HNM group and the BM group are illustrated in Fig. 3. The survival analysis curves for OS in the two groups are shown in Fig. S1. 
Table 3

Univariate analysis of factors affecting cancer-specific and overall survival

\begin{tabular}{|c|c|c|c|c|c|c|c|c|}
\hline \multirow[t]{3}{*}{ Factors } & \multicolumn{4}{|c|}{ 5-year Cancer-Specific Survival rate } & \multicolumn{4}{|c|}{ 5-year Overall Survival rate } \\
\hline & \multicolumn{4}{|c|}{$(\%$, mean $\pm S D)$} & \multicolumn{4}{|c|}{$(\%$, mean \pm SD $)$} \\
\hline & HNM & $\begin{array}{l}P \\
\text { value }\end{array}$ & BM & $\begin{array}{l}P \\
\text { value }\end{array}$ & HNM & $\begin{array}{l}P \\
\text { value }\end{array}$ & BM & $\begin{array}{l}P \\
\text { value }\end{array}$ \\
\hline Age (CSS/OS) & & $凶 0.001$ & & $凶 0.001$ & & 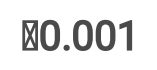 & & $凶 0.001$ \\
\hline$<=58 /<=63$ & $\begin{array}{l}91.5 \pm \\
0.5\end{array}$ & & $\begin{array}{l}94.8 \pm \\
0.2\end{array}$ & & $\begin{array}{l}88.5 \pm \\
0.5\end{array}$ & & $\begin{array}{l}92.7 \pm \\
0.2\end{array}$ & \\
\hline $59-78$ / 64-79 & $\begin{array}{l}88.3 \pm \\
0.5\end{array}$ & & $\begin{array}{l}91.6 \pm \\
0.3\end{array}$ & & $\begin{array}{l}75.7 \pm \\
0.8\end{array}$ & & $\begin{array}{l}81.7 \pm \\
0.4\end{array}$ & \\
\hline >=79 / >=80 & $\begin{array}{l}80.2 \pm \\
1.1\end{array}$ & & $\begin{array}{l}82.6 \pm \\
0.7\end{array}$ & & $\begin{array}{l}41.0 \pm \\
1.2\end{array}$ & & $\begin{array}{l}48.8 \pm \\
1.0\end{array}$ & \\
\hline Sex & & $\mathbb{\otimes 0 . 0 0 1}$ & & $\mathbb{\nabla 0 . 0 0 1}$ & & $凶 0.001$ & & $凶 0.001$ \\
\hline Male & $\begin{array}{l}86.9 \pm \\
0.4\end{array}$ & & $\begin{array}{l}90.5 \pm \\
0.2\end{array}$ & & $\begin{array}{l}73.3 \pm \\
0.6\end{array}$ & & $\begin{array}{l}82.7 \pm \\
0.3\end{array}$ & \\
\hline Female & $\begin{array}{l}90.6 \pm \\
0.6\end{array}$ & & $\begin{array}{l}94.8 \pm \\
0.2\end{array}$ & & $\begin{array}{l}77.2 \pm \\
0.9\end{array}$ & & $\begin{array}{l}89.7 \pm \\
0.2\end{array}$ & \\
\hline Race & & 0.011 & & 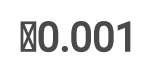 & & 0.366 & & $\triangle 0.001$ \\
\hline White & $\begin{array}{l}87.9 \pm \\
0.4\end{array}$ & & $\begin{array}{l}92.8 \pm \\
0.1\end{array}$ & & $\begin{array}{l}77.4 \pm \\
0.5\end{array}$ & & $\begin{array}{l}86.2 \pm \\
0.2\end{array}$ & \\
\hline a Others & $\begin{array}{l}82.3 \pm \\
4.7\end{array}$ & & $\begin{array}{l}80.2 \pm \\
1.8\end{array}$ & & $\begin{array}{l}71.5 \pm \\
5.4\end{array}$ & & $\begin{array}{l}73.5 \pm \\
2.0\end{array}$ & \\
\hline Histology & & $\mathbb{0 0 . 0 0 1}$ & & $\mathbb{\nabla 0 . 0 0 1}$ & & 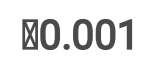 & & $凶 0.001$ \\
\hline NM & $\begin{array}{l}67.7 \pm \\
1.4\end{array}$ & & $\begin{array}{l}72.4 \pm \\
0.7\end{array}$ & & $\begin{array}{l}53.7 \pm \\
1.4\end{array}$ & & $\begin{array}{l}62.1 \pm \\
0.7\end{array}$ & \\
\hline LMM & $\begin{array}{l}96.1 \pm \\
0.4\end{array}$ & & $\begin{array}{l}97.7 \pm \\
0.3\end{array}$ & & $\begin{array}{l}80.2 \pm \\
0.8\end{array}$ & & $\begin{array}{l}86.2 \pm \\
0.8\end{array}$ & \\
\hline SMM & $\begin{array}{l}91.0 \pm \\
0.5\end{array}$ & & $\begin{array}{l}96.8 \pm \\
0.1\end{array}$ & & $\begin{array}{l}79.9 \pm \\
0.7\end{array}$ & & $\begin{array}{l}91.9 \pm \\
0.2\end{array}$ & \\
\hline
\end{tabular}

HNM, head and neck melanoma; BM, body melanoma.

SD, standard deviation; CSS, cancer-specific survival; OS, overall survival; NM, nodular melanoma; LMM, Lentigo maligna melanoma; SSM, superficial spreading melanoma.

a Others $=$ black, Asian or pacific islander, American indian/Alaska native.

b Other melanoma: International Classification of Diseases for Oncology-0-3, codes 8722-8723, 8730, 8740-8741, 8744-8746, 8761, 8770-8774, 8780. 


\begin{tabular}{|c|c|c|c|c|c|c|c|c|}
\hline \multirow[t]{3}{*}{ Factors } & \multicolumn{4}{|c|}{ 5-year Cancer-Specific Survival rate } & \multicolumn{4}{|c|}{ 5-year Overall Survival rate } \\
\hline & \multicolumn{4}{|c|}{$(\%$, mean $\pm S D)$} & \multicolumn{4}{|c|}{$(\%$, mean $\pm S D)$} \\
\hline & HNM & $\begin{array}{l}P \\
\text { value }\end{array}$ & BM & $\begin{array}{l}P \\
\text { value }\end{array}$ & HNM & $\begin{array}{l}P \\
\text { value }\end{array}$ & BM & $\begin{array}{l}P \\
\text { value }\end{array}$ \\
\hline $\begin{array}{l}\text { b Other } \\
\text { Melanoma }\end{array}$ & $\begin{array}{l}81.9 \pm \\
1.3\end{array}$ & & $\begin{array}{l}86.7 \pm \\
0.6\end{array}$ & & $\begin{array}{l}68.3 \pm \\
1.5\end{array}$ & & $\begin{array}{l}78.6 \pm \\
0.7\end{array}$ & \\
\hline Thickness & & $凶 0.001$ & & $凶 0.001$ & & $\triangle 0.001$ & & $\triangle 0.001$ \\
\hline$<0.6 \mathrm{~mm}$ & $\begin{array}{l}96.8 \pm \\
0.3\end{array}$ & & $\begin{array}{l}98.6 \pm \\
0.1\end{array}$ & & $\begin{array}{l}84.1 \pm \\
0.6\end{array}$ & & $\begin{array}{l}93.3 \pm \\
0.2\end{array}$ & \\
\hline $0.6-1.0 \mathrm{~mm}$ & $\begin{array}{l}93.2 \pm \\
0.7\end{array}$ & & $\begin{array}{l}97.4 \pm \\
0.2\end{array}$ & & $\begin{array}{l}79.6 \pm \\
1.1\end{array}$ & & $\begin{array}{l}92.2 \pm \\
0.3\end{array}$ & \\
\hline $1.01-2.0 \mathrm{~mm}$ & $\begin{array}{l}85.9 \pm \\
1.0\end{array}$ & & $\begin{array}{l}91.6 \pm \\
0.4\end{array}$ & & $\begin{array}{l}72.8 \pm \\
1.2\end{array}$ & & $\begin{array}{l}85.6 \pm \\
0.5\end{array}$ & \\
\hline $2.01-4.0 \mathrm{~mm}$ & $\begin{array}{l}73.2 \pm \\
1.5\end{array}$ & & $\begin{array}{l}78.5 \pm \\
0.8\end{array}$ & & $\begin{array}{l}58.8 \pm \\
1.6\end{array}$ & & $\begin{array}{l}68.2 \pm \\
0.9\end{array}$ & \\
\hline$>4.0 \mathrm{~mm}$ & $\begin{array}{l}58.6 \pm \\
1.8\end{array}$ & & $\begin{array}{l}57.5 \pm \\
1.1\end{array}$ & & $\begin{array}{l}45.1 \pm \\
1.7\end{array}$ & & $\begin{array}{l}46.0 \pm \\
1.1\end{array}$ & \\
\hline Ulceration & & $凶 0.001$ & & $凶 0.001$ & & $凶 0.001$ & & $\triangle 0.001$ \\
\hline No & $\begin{array}{l}92.2 \pm \\
0.3\end{array}$ & & $\begin{array}{l}96.5 \pm \\
0.1\end{array}$ & & $\begin{array}{l}79.1 \pm \\
0.5\end{array}$ & & $\begin{array}{l}90.9 \pm \\
0.2\end{array}$ & \\
\hline Yes & $\begin{array}{l}64.3 \pm \\
1.4\end{array}$ & & $\begin{array}{l}69.1 \pm \\
0.7\end{array}$ & & $\begin{array}{l}50.3 \pm \\
1.4\end{array}$ & & $\begin{array}{l}58.5 \pm \\
0.7\end{array}$ & \\
\hline Stage & & $凶 0.001$ & & $凶 0.001$ & & $凶 0.001$ & & $凶 0.001$ \\
\hline Localized & $\begin{array}{l}92.7 \pm \\
0.3\end{array}$ & & $\begin{array}{l}96.4 \pm \\
0.1\end{array}$ & & $\begin{array}{l}78.7 \pm \\
0.5\end{array}$ & & $\begin{array}{l}90.0 \pm \\
0.2\end{array}$ & \\
\hline Regional & $\begin{array}{l}61.7 \pm \\
1.6\end{array}$ & & $\begin{array}{l}65.5 \pm \\
0.9\end{array}$ & & $\begin{array}{l}51.0 \pm \\
1.6\end{array}$ & & $\begin{array}{l}58.2 \pm \\
0.9\end{array}$ & \\
\hline Distant & $\begin{array}{l}36.6 \pm \\
4.2\end{array}$ & & $\begin{array}{l}30.5 \pm \\
2.4\end{array}$ & & $\begin{array}{l}28.4 \pm \\
3.8\end{array}$ & & $\begin{array}{l}26.0 \pm \\
2.3\end{array}$ & \\
\hline Metastases & & $\nabla 0.001$ & & $\mathbb{\nabla 0 . 0 0 1}$ & & $凶 0.001$ & & $\triangle 0.001$ \\
\hline
\end{tabular}

HNM, head and neck melanoma; BM, body melanoma.

SD, standard deviation; CSS, cancer-specific survival; OS, overall survival; NM, nodular melanoma; LMM, Lentigo maligna melanoma; SSM, superficial spreading melanoma.

${ }^{\text {a }}$ Others $=$ black, Asian or pacific islander, American indian/Alaska native .

b Other melanoma: International Classification of Diseases for Oncology-0-3, codes 8722-8723, 8730, 8740-8741, 8744-8746, 8761, 8770-8774, 8780. 


\begin{tabular}{|c|c|c|c|c|c|c|c|c|}
\hline \multirow[t]{3}{*}{ Factors } & \multicolumn{4}{|c|}{ 5-year Cancer-Specific Survival rate } & \multicolumn{4}{|c|}{ 5-year Overall Survival rate } \\
\hline & \multicolumn{4}{|c|}{$(\%$, mean $\pm S D)$} & \multicolumn{4}{|c|}{$(\%$, mean \pm SD $)$} \\
\hline & HNM & $\begin{array}{l}\mathrm{P} \\
\text { value }\end{array}$ & BM & $\begin{array}{l}P \\
\text { value }\end{array}$ & HNM & $\begin{array}{l}\mathrm{P} \\
\text { value }\end{array}$ & BM & $\begin{array}{l}P \\
\text { value }\end{array}$ \\
\hline No & \multicolumn{2}{|l|}{$\begin{array}{l}88.7 \pm \\
0.4\end{array}$} & \multicolumn{2}{|l|}{$\begin{array}{l}93.2 \pm \\
0.1\end{array}$} & \multicolumn{2}{|l|}{$\begin{array}{l}75.1 \pm \\
0.5\end{array}$} & \multicolumn{2}{|l|}{$\begin{array}{l}86.7 \pm \\
0.2\end{array}$} \\
\hline Yes & \multicolumn{2}{|l|}{$\begin{array}{l}30.1 \pm \\
4.5\end{array}$} & \multicolumn{2}{|l|}{$\begin{array}{l}20.3 \pm \\
2.4\end{array}$} & \multicolumn{2}{|l|}{$\begin{array}{l}23.8 \pm \\
4.2\end{array}$} & \multicolumn{2}{|l|}{$\begin{array}{l}17.6 \pm \\
2.2\end{array}$} \\
\hline \multicolumn{2}{|l|}{ Surgery } & \multicolumn{2}{|l|}{0.002} & 0.061 & \multicolumn{3}{|c|}{$凶 0.001$} & $凶 0.001$ \\
\hline No & $\begin{array}{l}82.0 \pm \\
3.0\end{array}$ & & $\begin{array}{l}91.6 \pm \\
1.2\end{array}$ & & $\begin{array}{l}60.8 \pm \\
3.8\end{array}$ & & $\begin{array}{l}80.7 \pm \\
1.7\end{array}$ & \\
\hline Yes & $\begin{array}{l}88.0 \pm \\
0.4\end{array}$ & & $\begin{array}{l}92.6 \pm \\
0.2\end{array}$ & & $\begin{array}{l}74.7 \pm \\
0.5\end{array}$ & & $\begin{array}{l}86.1 \pm \\
0.2\end{array}$ & \\
\hline \multicolumn{9}{|c|}{ HNM, head and neck melanoma; BM, body melanoma. } \\
\hline \multicolumn{9}{|c|}{$\begin{array}{l}\text { SD, standard deviation; CSS, cancer-specific survival; OS, overall survival; NM, nodular melanoma; LMM, } \\
\text { Lentigo maligna melanoma; SSM, superficial spreading melanoma. }\end{array}$} \\
\hline \multicolumn{9}{|c|}{ a Others = black, Asian or pacific islander, American indian/Alaska native. } \\
\hline $\begin{array}{l}\mathrm{b} \text { Other m } \\
8740-87\end{array}$ & $\begin{array}{l}\text { : Intern } \\
-8746,\end{array}$ & $\begin{array}{l}\text { al Clas } \\
1,8770\end{array}$ & $\begin{array}{l}\text { cation } \\
74,87\end{array}$ & iseases & cology-c & $\operatorname{codes} 8$ & $22-872$ & 730, \\
\hline
\end{tabular}


Table 4

Multivariate analysis of factors affecting cancer-specific and overall survival

Factors
Cancer specific survival

$\mathrm{HR}(95 \% \mathrm{Cl})$

HNM

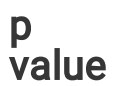

BM

Reference

value

\section{Overall survival}

$\operatorname{HR}(95 \% \mathrm{Cl})$

HNM

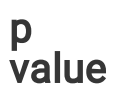

BM

value

\section{Age}

(CSS/OS)

\begin{tabular}{|c|c|c|c|c|c|c|c|c|}
\hline $\begin{array}{l}<=58 / \\
<=63\end{array}$ & Reference & & Reference & & Reference & & Referenc & \\
\hline $\begin{array}{l}59-78 / \\
64-79\end{array}$ & $\begin{array}{l}1.4 \\
(1.213- \\
1.615)\end{array}$ & $\begin{array}{l}<.001 \\
\end{array}$ & $\begin{array}{l}1.402 \\
(1.290- \\
1.523)\end{array}$ & $\begin{array}{l}<.001 \\
0.00\end{array}$ & $\begin{array}{l}2.571 \\
(2.319- \\
2.850)\end{array}$ & <. 001 & $\begin{array}{l}2.743 \\
(2.583- \\
2.913)\end{array}$ & $\dot{0} 001$ \\
\hline $\begin{array}{l}>=79 / \\
>=80\end{array}$ & $\begin{array}{l}2.219 \\
(1.892- \\
2.602)\end{array}$ & $\begin{array}{l}<.001 \\
\end{array}$ & $\begin{array}{l}2.487 \\
(2.237- \\
2.765)\end{array}$ & $\begin{array}{l}< \\
0.001\end{array}$ & $\begin{array}{l}7.668 \\
(6.918- \\
8.498)\end{array}$ & <. 001 & $\begin{array}{l}8.028 \\
(7.516- \\
8.575)\end{array}$ & $\begin{array}{l}<.001 \\
\end{array}$ \\
\hline
\end{tabular}

\section{Sex}

\begin{tabular}{|c|c|c|c|c|c|c|c|c|}
\hline Male & Reference & & Reference & & Reference & & Reference & \\
\hline Female & $\begin{array}{l}0.789 \\
(0.686- \\
0.908)\end{array}$ & 0.001 & $\begin{array}{l}0.694 \\
(0.642- \\
0.750)\end{array}$ & $\begin{array}{l}< \\
0.001\end{array}$ & $\begin{array}{l}0.854 \\
(0.784- \\
0.930)\end{array}$ & $\begin{array}{l}< \\
0.001\end{array}$ & $\begin{array}{l}0.663 \\
(0.629- \\
0.698)\end{array}$ & $\begin{array}{l}< \\
0.001\end{array}$ \\
\hline
\end{tabular}

\section{Histology}

\begin{tabular}{|c|c|c|c|c|c|c|c|c|}
\hline NM & Reference & & Reference & & Reference & & Referenc & \\
\hline LMM & $\begin{array}{l}0.534 \\
(0.429- \\
0.665)\end{array}$ & $\begin{array}{l}<.001 \\
0.00\end{array}$ & $\begin{array}{l}0.557 \\
(0.421- \\
0.737)\end{array}$ & $\begin{array}{l}< \\
0.001\end{array}$ & $\begin{array}{l}0.794 \\
(0.700- \\
0.901)\end{array}$ & $<.001$ & $\begin{array}{l}0.984 \\
(0.876- \\
1.106)\end{array}$ & 0.787 \\
\hline SMM & $\begin{array}{l}0.834 \\
(0.712- \\
0.978)\end{array}$ & 0.025 & $\begin{array}{l}0.611 \\
(0.552- \\
0.675)\end{array}$ & $\begin{array}{l}< \\
0.001\end{array}$ & $\begin{array}{l}0.851 \\
(0.760- \\
0.953)\end{array}$ & 0.005 & $\begin{array}{l}0.723 \\
(0.672- \\
0.777)\end{array}$ & $\begin{array}{l}< \\
0.001\end{array}$ \\
\hline $\begin{array}{l}\text { a Other } \\
\text { Melanoma }\end{array}$ & $\begin{array}{l}0.606 \\
(0.512- \\
0.718)\end{array}$ & $<.001$ & $\begin{array}{l}0.812 \\
(0.731- \\
0.902)\end{array}$ & $\begin{array}{l}< \\
0.001\end{array}$ & $\begin{array}{l}0.655 \\
(0.579- \\
0.739)\end{array}$ & $<.001$ & $\begin{array}{l}0.811 \\
(0.748- \\
0.878)\end{array}$ & $\begin{array}{l}< \\
0.001\end{array}$ \\
\hline
\end{tabular}

\section{Thickness}

$<0.6 \mathrm{~mm} \quad$ Reference Reference Reference Rence

HNM, head and neck melanoma; BM, body melanoma.

CSS, cancer-specific survival; OS, overall survival; NM, nodular melanoma; LMM, Lentigo maligna melanoma; SSM, superficial spreading melanoma.

a Other melanoma: International Classification of Diseases for Oncology-0-3, codes 8722-8723, 8730, 8740-8741, 8744-8746, 8761, 8770-8774, 8780. 


\begin{tabular}{|c|c|c|c|c|c|c|c|c|}
\hline \multirow[t]{3}{*}{ Factors } & \multicolumn{4}{|c|}{ Cancer specific survival } & \multicolumn{4}{|c|}{ Overall survival } \\
\hline & \multicolumn{4}{|c|}{$\mathrm{HR}(95 \% \mathrm{Cl})$} & \multicolumn{4}{|c|}{$\mathrm{HR}(95 \% \mathrm{Cl})$} \\
\hline & HNM & value & BM & value & HNM & $\begin{array}{l}\mathrm{p} \\
\text { value }\end{array}$ & BM & $\begin{array}{l}\text { p } \\
\text { value }\end{array}$ \\
\hline $0.6-1.0 \mathrm{~mm}$ & $\begin{array}{l}1.845 \\
(1.446- \\
2.354)\end{array}$ & $\dot{0} 001$ & $\begin{array}{l}1.802 \\
(1.520- \\
2.137)\end{array}$ & $\begin{array}{l}< \\
0.001\end{array}$ & $\begin{array}{l}1.232 \\
(1.093- \\
1.389)\end{array}$ & 0.001 & $\begin{array}{l}1.159 \\
(1.066- \\
1.259)\end{array}$ & 0.001 \\
\hline $\begin{array}{l}1.01- \\
2.0 \mathrm{~mm}\end{array}$ & $\begin{array}{l}3.285 \\
(2.643- \\
4.082)\end{array}$ & $\hat{0} .001$ & $\begin{array}{l}3.171 \\
(2.718- \\
3.698)\end{array}$ & $\hat{0} .001$ & $\begin{array}{l}1.557 \\
(1.383- \\
1.753)\end{array}$ & $\hat{0} .001$ & $\begin{array}{l}1.443 \\
(1.327- \\
1.569)\end{array}$ & $\hat{0.001}$ \\
\hline $\begin{array}{l}2.01- \\
4.0 \mathrm{~mm}\end{array}$ & $\begin{array}{l}3.942 \\
(3.139- \\
4.951)\end{array}$ & $\hat{0}_{0.001}$ & $\begin{array}{l}4.328 \\
(3.683- \\
5.086)\end{array}$ & <. 001 & $\begin{array}{l}1.767 \\
(1.551- \\
2.014)\end{array}$ & $\begin{array}{l}<.001 \\
0.001\end{array}$ & $\begin{array}{l}1.951 \\
(1.780- \\
2.140)\end{array}$ & $\begin{array}{l}<.001 \\
0\end{array}$ \\
\hline$>4.0 \mathrm{~mm}$ & $\begin{array}{l}4.403 \\
(3.428- \\
5.655)\end{array}$ & $\hat{0}_{0.001}$ & $\begin{array}{l}5.535 \\
(4.672- \\
6.558)\end{array}$ & $\hat{0}_{0.001}$ & $\begin{array}{l}2.078 \\
(1.787- \\
2.417)\end{array}$ & $\begin{array}{l}<.001 \\
0.001\end{array}$ & $\begin{array}{l}2.513 \\
(2.270- \\
2.781)\end{array}$ & $\begin{array}{l}<.001 \\
\end{array}$ \\
\hline \multicolumn{9}{|l|}{ Ulceration } \\
\hline No & \multicolumn{2}{|l|}{ Reference } & \multicolumn{2}{|c|}{ Reference } & \multicolumn{2}{|l|}{ Reference } & \multicolumn{2}{|l|}{ Reference } \\
\hline Yes & $\begin{array}{l}1.843 \\
(1.615- \\
2.103)\end{array}$ & $\begin{array}{l}< \\
0.001\end{array}$ & $\begin{array}{l}2.235 \\
(2.045- \\
2.443)\end{array}$ & <. 001 & $\begin{array}{l}1.472 \\
(1.340- \\
1.618)\end{array}$ & $\hat{0} .001$ & $\begin{array}{l}1.778 \\
(1.668- \\
1.895)\end{array}$ & $\begin{array}{l}<.001 \\
0.00\end{array}$ \\
\hline \multicolumn{9}{|l|}{ Stage } \\
\hline Localized & \multicolumn{2}{|l|}{ Reference } & \multicolumn{2}{|c|}{ Reference } & \multicolumn{2}{|l|}{ Reference } & \multicolumn{2}{|l|}{ Reference } \\
\hline Regional & $\begin{array}{l}2.547 \\
(2.196- \\
2.954)\end{array}$ & $\hat{0} .001$ & $\begin{array}{l}3.203 \\
(2.923- \\
3.509)\end{array}$ & $\hat{0} .001$ & $\begin{array}{l}1.677 \\
(1.501- \\
1.873)\end{array}$ & $\hat{0} .001$ & $\begin{array}{l}2.049 \\
(1.913- \\
2.195)\end{array}$ & $\begin{array}{l}<.001 \\
\end{array}$ \\
\hline Distant & $\begin{array}{l}3.686 \\
(2.015- \\
6.744)\end{array}$ & $\hat{0} .001$ & $\begin{array}{l}5.287 \\
(4.097- \\
6.824)\end{array}$ & $\begin{array}{l}< \\
0.001\end{array}$ & $\begin{array}{l}2.434 \\
(1.523- \\
3.891)\end{array}$ & $<.001$ & $\begin{array}{l}3.679 \\
(2.941- \\
4.602)\end{array}$ & $\begin{array}{l}<.001 \\
0.00\end{array}$ \\
\hline \multicolumn{9}{|l|}{ Metastases } \\
\hline No & \multicolumn{2}{|l|}{ Reference } & \multicolumn{2}{|c|}{ Reference } & \multicolumn{2}{|l|}{ Reference } & \multicolumn{2}{|l|}{ Reference } \\
\hline Yes & $\begin{array}{l}2.323 \\
(1.237- \\
4.363)\end{array}$ & 0.009 & $\begin{array}{l}2.540 \\
(1.935- \\
3.335)\end{array}$ & $\begin{array}{l}<.001 \\
0.01\end{array}$ & $\begin{array}{l}2.061 \\
(1.244- \\
3.413)\end{array}$ & 0.005 & $\begin{array}{l}2.273 \\
(1.777- \\
2.908)\end{array}$ & $\begin{array}{l}<.001 \\
0.00\end{array}$ \\
\hline \multicolumn{9}{|c|}{ HNM, head and neck melanoma; BM, body melanoma. } \\
\hline \multicolumn{9}{|c|}{$\begin{array}{l}\text { CSS, cancer-specific survival; OS, overall survival; NM, nodular melanoma; LMM, Lentigo maligna } \\
\text { melanoma; SSM, superficial spreading melanoma. }\end{array}$} \\
\hline
\end{tabular}




\begin{tabular}{|c|c|c|c|c|c|c|c|c|}
\hline \multirow[t]{3}{*}{ Factors } & \multicolumn{4}{|c|}{ Cancer specific survival } & \multicolumn{4}{|c|}{ Overall survival } \\
\hline & \multicolumn{4}{|c|}{$\mathrm{HR}(95 \% \mathrm{Cl})$} & \multicolumn{4}{|c|}{$\mathrm{HR}(95 \% \mathrm{Cl})$} \\
\hline & HNM & value & BM & value & HNM & $\stackrel{p}{\text { value }}$ & BM & $\begin{array}{l}\text { p } \\
\text { value }\end{array}$ \\
\hline \multicolumn{9}{|l|}{ Surgery } \\
\hline No & \multicolumn{2}{|l|}{ Reference } & \multicolumn{2}{|c|}{ Reference } & \multicolumn{2}{|c|}{ Reference } & \multicolumn{2}{|c|}{ Reference } \\
\hline Yes & $\begin{array}{l}0.370 \\
(0.263- \\
0.520)\end{array}$ & $\hat{0.001}$ & $\begin{array}{l}0.538 \\
(0.411- \\
0.705)\end{array}$ & $<.001$ & $\begin{array}{l}0.568 \\
(0.457- \\
0.707)\end{array}$ & $\dot{0} 001$ & $\begin{array}{l}0.511 \\
(0.433- \\
0.604)\end{array}$ & $\dot{\delta} 001$ \\
\hline \multicolumn{9}{|c|}{ HNM, head and neck melanoma; BM, body melanoma. } \\
\hline \multicolumn{9}{|c|}{$\begin{array}{l}\text { CSS, cancer-specific survival; OS, overall survival; NM, nodular melanoma; LMM, Lentigo maligna } \\
\text { melanoma; SSM, superficial spreading melanoma. }\end{array}$} \\
\hline
\end{tabular}

CSS = cancer-specific survival; HNM = head and neck melanoma; $\mathrm{BM}=$ body melanoma

\subsection{Nomogram development}

In this study, eight independent prognostic factors were included to construct nomograms for HNM patients or BM patients to predict 3-, 5-, and 10-year CSS and OS (Fig. 4).

$(a, c)$ predicting CSS and OS for HNM training cohort; $(b, d)$ predicting CSS and OS for BM training cohort.

CSS = cancer-specific survival; OS = overall survival; $\mathrm{HNM}=$ head and neck melanoma;

$\mathrm{BM}=$ body melanoma .

The nomogram of HNM showed that thickness $(>4.0 \mathrm{~mm})$, which had the largest absolute values, was the strongest contributor to the risk of CSS prognosis, followed by distant stage, no surgery, having metastases and age $>79$ years. Notably, thickness $(>4.0 \mathrm{~mm}$ ) also contributed to the highest CSS risk in the BM nomogram, but distant stage had similar points. Present metastases, age over 79 years and ulceration were strong factors after distant stage in the BM nomogram of CSS.

By adding the scores of each predictor based on individual conditions (Table 5), the HNM nomogram and BM nomogram can be used to predict the probability of 3-, 5- and 10-year CSS and OS rates for HNM patients and BM patients, respectively. For example, Patient $\mathrm{A}$ is a 65-year-old male patient who had a head (scalp) tumor thickness of $3.0 \mathrm{~mm}$ and was diagnosed with localized SSM without ulceration and metastases and then underwent surgery. A total of 161.38 points were given to this patient, and the estimated 10 -year CSS rate was $77 \%$. Patient $B$, who had all the same clinical features as patient $A$ and underwent surgery but had a 
tumor site on the body (shoulder), had a total of 132.03 points. Eventually, this patient's predicted 10-year CSS was $87 \%$, which was $10 \%$ higher than the CSS rate of patient $A$. 
Table 5

Detailed scores of predictors in nomograms.

\begin{tabular}{|c|c|c|c|c|}
\hline & \multicolumn{2}{|c|}{ Cancer-specific survival nomogram } & \multicolumn{2}{|c|}{ Overall survival nomogram } \\
\hline & HNM & BM & HNM & BM \\
\hline Total Points & 500 & 450 & 300 & 350 \\
\hline \multicolumn{5}{|l|}{ Age (CSS/OS) } \\
\hline$<=58 /<=63$ & 0 & 0 & 0 & 0 \\
\hline $59-78 / 64-79$ & 22.72 & 19.7 & 46.27 & 48.34 \\
\hline$>=79 />=80$ & 53.84 & 53.35 & 100 & 100 \\
\hline \multicolumn{5}{|l|}{ Sex } \\
\hline Male & 16.01 & 21.38 & 7.73 & 19.71 \\
\hline Female & 0 & 0 & 0 & 0 \\
\hline \multicolumn{5}{|l|}{ Histology } \\
\hline NM & 42.38 & 34.26 & 20.88 & 15.56 \\
\hline LMM & 0 & 0 & 9.54 & 14.75 \\
\hline SSM & 30.1 & 5.44 & 12.91 & 0 \\
\hline a Other Melanoma & 8.48 & 22.09 & 0 & 5.48 \\
\hline \multicolumn{5}{|l|}{ Thickness } \\
\hline$<0.6 \mathrm{~mm}$ & 0 & 0 & 0 & 0 \\
\hline $0.6-1.0 \mathrm{~mm}$ & 41.26 & 34.39 & 10.2 & 7.03 \\
\hline $1.01-2.0 \mathrm{~mm}$ & 80.2 & 67.33 & 21.67 & 17.52 \\
\hline $2.01-4.0 \mathrm{~mm}$ & 92.55 & 85.51 & 27.93 & 31.99 \\
\hline$>4.0 \mathrm{~mm}$ & 100 & 100 & 35.92 & 44.23 \\
\hline \multicolumn{5}{|l|}{ Ulceration } \\
\hline No & 0 & 0 & 0 & 0 \\
\hline Yes & 41.37 & 47.07 & 19.01 & 27.64 \\
\hline
\end{tabular}

HNM, head and neck melanoma; BM, body melanoma.

CSS, cancer-specific survival; OS, overall survival; NM, nodular melanoma; LMM, Lentigo maligna melanoma; SSM, superficial spreading melanoma.

a Other melanoma: International Classification of Diseases for Oncology-0-3, codes 8722-8723, 8730, 8740-8741, 8744-8746, 8761, 8770-8774, 8780. 


\begin{tabular}{lllll} 
Stage & & & & \\
Localized & 0 & 0 & 25.38 & 34.43 \\
\hline Regional & 63.22 & 68.11 & 43.79 & 62.69 \\
\hline Distant & 88.3 & 97.55 & & \\
\hline Metastases & & & 0 & 0 \\
\hline No & 0 & 0 & 35.71 & 39.65 \\
\hline Yes & 57.31 & 54.93 & & \\
\hline Surgery & & & 27.83 & 32.28 \\
\hline No & 67.46 & 36.21 & 0 & 0 \\
\hline Yes & 0 & 0 & 0 & \\
\hline
\end{tabular}

HNM, head and neck melanoma; BM, body melanoma.

CSS, cancer-specific survival; OS, overall survival; NM, nodular melanoma; LMM, Lentigo maligna melanoma; SSM, superficial spreading melanoma.

a Other melanoma: International Classification of Diseases for Oncology-0-3, codes 8722-8723, 8730, 8740-8741, 8744-8746, 8761, 8770-8774, 8780.

\subsection{Validation and calibration of the nomogram}

The C-index and AUC values were used to evaluate the accuracy and discriminative ability of the nomogram. The C-index of the CSS prediction nomogram for HNM patients was $0.839 \pm 0.005$ in the training cohort and $0.848 \pm 0.008$ in the validation cohort. The C-index of the CSS nomogram for the BM group were $0.895 \pm$ 0.003 and $0.888 \pm 0.004$ in the training cohort and the validation cohort, respectively. In addition, the C-index of OS prediction for HNM patients and BM patients in the training cohort were $0.785 \pm 0.004$ and $0.841 \pm$ 0.002 , respectively; in the validation cohort, they were $0.796 \pm 0.006$ and $0.840 \pm 0.003$, respectively.

The AUCs for the 3-, 5- and 10-year CSS rates in the training cohort of the HNM group were 0.871, 0.865 and 0.854 , and those in the validation cohort were $0.881,0.879$ and 0.861 , respectively. For predicting the $3-, 5-$ and 10-year CSS rates in the BM population, the AUCs were 0.924, 0.918 and 0.901 in the training cohort and $0.916,0.908$ and 0.893 in the validation cohort, respectively (Fig. 5). The AUCs for the OS rates in different cohorts are illustrated in Fig. 5.

a-d predicted 3-, 5- and 10-year CSS rates for HNM training and validation cohort, BM training and validation cohort, respectively. e-h predicted 3-, 5- and 10-year OS rates for HNM training and validation cohort, BM training and validation cohort, respectively.

The calibration plots for the probability of 3-, 5- and 10-year CSS showed great consistency between the nomogram-based prediction and the actual observed outcomes (Fig. 6). 
The calibration curves predicted CSS of patients with HNM in the training cohort (a-c) and in the validation cohort (d-f). The calibration curves predicted CSS of patients with BM in the training cohort $(g-h)$ and in the validation cohort (j-I).

Similar results were found in the calibration of the OS prediction nomograms (Fig. S2). These results indicated that both the HNM nomogram and the BM nomogram exhibit excellent performance for predicting the survival of malignant cutaneous melanoma patients.

\section{Discussion}

In this study, we compared the characteristics of HNM patients with those of BM patients based on the data of a large SEER population. Then, we performed survival analysis of the HNM and BM groups to construct nomograms for individualized survival prediction

Hoersch et al[16] compared 844 patients with HNM and 4858 patients with BM and performed univariate and multivariate analyses, but they found no OS differences between patients with HNM and patients with BM, and the identified significant prognostic factors of HNM were inconsistent with those identified in our study. The low number of cases and different populations in that study could have led to the controversial results. Kevin et al[14] performed a comparison of 4561 pediatric patients with HNM or BM, but they did not study prognostic factors. Previous studies have identified significant independent prognostic factors of melanoma, and some nomograms were developed for predicting the OS or CSS of nonmetastatic melanoma patients, melanoma patients with brain metastases or nodular melanoma patients[6, 24-27]. To date, a specific nomogram for HNM or BM has not been developed.

A total of 70605 eligible patients in the United States from 2004 to 2015 were identified in our study. To our knowledge, this was the largest population-based study to perform a comprehensive comparison between $\mathrm{HNM}$ and $\mathrm{BM}$, including their characteristics and survival. Furthermore, this is the first time that high-quality nomograms have been constructed for predicting the prognosis of HNM patients and BM patients, respectively.

HNM patients accounted for $21 \%$ of all eligible patients in our study, which was consistent with that in previous reports $[9,16]$. According to the univariate prognostic factor analysis of all eligible patients, significant differences were seen between patients with HNM and patients with BM. Notably, the 5-year OS rate of HNM patients was over $10 \%$ less than that of BM patients. The relatively high frequency and poorer prognosis of HNM suggest that cutaneous melanoma in head and neck areas requires more attention and health management.

Both the mean and median age of patients with HNM were greater than those of BM patients. HNM was found more than twice as frequently as BM in elderly patients (age $>=79$ ). The finding that HNM occurred more frequently in elderly individuals was also observed in other population-based studies[7, 28-30]. Elderly individuals usually suffer longer chronic sun exposure than younger individuals in regions of the head and neck, explaining the higher melanoma rate. In addition, based on the subgroup analysis of age, we observed that the 5-year CSS and OS rates were lower for patients with HNM than for those with BM. 
In accordance with the findings of previous studies[15, 31-33], we found significant sex differences between HNM and BM. Nearly three-quarters of patients with HNM were male, while the percentage of females was close to that of males in the BM population in our study. Usually, women are more active in the prevention of sun exposure on the head and neck, which may reduce the risk of HNM[8]. Females showed a better prognosis than males in both the HNM and BM groups in our study. Interestingly, we observed that the 5-year OS of male patients with BM was better than that of female patients with HNM. This result suggested the important role of head and neck regions.

SSM was the most common histologic subtype of melanoma in both the HNM and BM cohorts, but a higher proportion of SSM was noticed in the latter population. Notably, $31.8 \%$ of HNM patients were diagnosed with LMM, while only $6.6 \%$ of BM patients had LMM. Other population-based melanoma studies also found that a high percentage of LMM occurred on the head and neck[7, 9]. LMM has a prolonged radial growth phase before entering the vertical phase to invade the dermis[34]. Chronic sun damage to the head and neck may serve as an adverse factor in its evolution. Nodular melanoma is associated with worse prognosis than other histological subtypes[35]. Similarly, our study found that this subtype had the worst prognosis in both the HNM and BM populations. Notably, nodular melanoma on the head and neck showed markedly worse 5-year CSS and OS rates. SSM is thought to be a tumor with a better prognosis[36]. Additionally, SSM on the head and neck had a much poorer prognosis than SSM on other sites.

Previous studies $[9,14,15,37]$ reported that thicker tumors (more than $2.0 \mathrm{~mm}$ ) were more common on the head and neck, and this result is consistent with the findings of our study. The capillary vessels and lymphatic drainage systems in these areas are rich and complex, and these specialties may facilitate the growth of tumors. In addition, more ulcerations, distant stages and metastases were found in patients with HNM, which may also be related to the complex drainage systems of the head and neck. Thickness has been identified as a prognostic factor of melanoma, and increasing thickness implies worse prognosis[15]; these results were in agreement with those of our study. As thin malignant melanomas account for a high number of melanoma fatalities owing to their large proportion[23], we divided thin melanomas $(<=1.0 \mathrm{~mm}$ ) into two subgroups: $<0.6 \mathrm{~mm}$ and 0.6-1.0 $\mathrm{mm}$. We observed that when compared with the $<0.6 \mathrm{~mm}$ group, the latter subgroup did not show a significant impact on the 5-year survival of patients with BM but decreased the survival rate of HNM patients.

Ulceration, lymph node stage and metastasis have been identified as significant independent factors of cutaneous melanoma in previous studies and were also identified in our study[26, 27]. Notably, when presenting distant metastasis, compared to HNM patients, BM patients had worse 5-year CSS and OS rates.

Furthermore, we compared the treatment differences between patients with HNM and patients with BM. The percentage of HNM patients who underwent surgery was slightly lower, while their radiation rate was higher than that of BM patients. We did not find differences in the proportion of patients who received chemotherapy in these two groups.

Multivariate Cox proportional hazards analysis resulted in a model of HNM with age, sex, histology, thickness, ulceration, lymph node stage, metastases and surgery as independent prognostic variables. The same factors contributed to the model of BM. Furthermore, for convenient utilization of the models in clinical 
practice, we developed two nomograms to estimate the individual CSS and OS of patients with HNM, and we constructed another two nomograms for patients with BM.

The nomogram of HNM showed excellent predictive and discriminatory power in predicting CSS, with a Cindex of 0.839 (95\% confidence interval $(\mathrm{Cl}), 0.834-0.844)$ in the training cohort and $0.848(95 \% \mathrm{Cl}, 0.840-$ $0.856)$ in the validation cohort. Similarly, the CSS nomogram for BM had high C-indexes of $0.895(95 \% \mathrm{Cl}$, $0.892-0.898)$ in the training cohort and $0.888(95 \% \mathrm{Cl}, 0.884-0.892)$ in the validation cohort. The range of AUC values for the CSS nomograms was $0.854-0.924$, indicating that our models had great discriminatory ability. In addition, the calibration curves in our study indicated satisfactory consistency between the actual observations and the predicted values. The OS nomograms for HNM and BM also showed good performance for prognosis prediction.

This study has the following limitations that need to be acknowledged. First, we grouped melanoma on the head and neck together as HNM for analysis and did not investigate specific anatomic subsites in this region individually. A similar problem existed in the BM analysis. Previous studies suggested that some precise areas had worse cutaneous melanoma-specific prognoses, such as the scalp and breast[3, 5]. Second, our patients were limited to the SEER program. Although the database covers approximately $28 \%$ of the US population from 18 registries, the results of our study might not be generalizable to other regional groups, and future studies are needed to externally validate the proposed nomograms. Third, we could not obtain precise data on radiotherapy and chemotherapy from the SEER database, as patients only had "Yes" or "None/Unknown" labels for these variables; thus, only surgery was included in the survival analysis. Finally, as a retrospective study, it is susceptible to some inherent biases.

\section{Conclusion}

In summary, we compared the characteristics and survival of HNM and BM, and constructed independent nomograms for HNM and BM patients to facilitate individual health management.

\section{Declarations}

Ethics approval and consent to participate: As all the data of this study were derived from the SEER database, institutional review board approval and consent to participate were not demanded.

Consent for publication: Not applicable.

Availability of data and material: The data were collected from the Surveillance, Epidemiology, and End Results (SEER) database (URL: https://seer.cancer.gov/, Accession username:12814-Nov2019).

Conflicts of interest: The authors have no conflicts of interest to declare.

Funding : This work was supported by the National Natural Science Foundation of China区No.81972959; No.81972955区 
Authors' contributions: LZF and DYX conceived and designed the study idea. DYX and JRY performed data mining. DYX, CYH and YXS performed data statistical analyses. DYX, JRY and LHSC prepared figures and tables. DYX and JRY drafted the initial manuscript. JJ and WXJ reviewed and edited the initial manuscript. ZXY, XJL and XPP participated in the coordination of the study and reviewed the manuscript. LZF had primary responsibility for the final content. All authors have approved the final version of the manuscript.

Acknowledgements: Not applicable.

\section{Abbreviations}

head and neck melanoma (HNM)

body melanoma (BM)

Surveillance, Epidemiology, and End Results (SEER)

the concordance index (C-index)

the receiver operator characteristic (ROC) curve (AUC)

overall survival (OS)

cancer-specific survival (CSS)

standard deviations (SDs)

American Joint Committee on Cancer (AJCC)

Superficial spreading melanoma (SSM)

lentigo maligna melanoma (LMM)

\section{References}

1. Schadendorf D, van Akkooi ACJ, Berking C, Griewank KG, Gutzmer R, Hauschild A et al. Melanoma. The Lancet. 2018;392(10151):971-84. doi:10.1016/s0140-6736(18)31559-9.

2. Siegel RL, Miller KD, Jemal A. Cancer statistics, 2020. CA Cancer J Clin. 2020;70(1):7-30. doi:10.3322/caac.21590.

3. Garbe C, Büttner P, Bertz J, Burg G, d'Hoedt B, Drepper H et al. Primary cutaneous melanoma. Prognostic classification of anatomic location. 1995;75(10):2492-8. doi:10.1002/10970142(19950515)75:10<2492::aid-cncr2820751015>3.0.co;2-w.

4. Callender G, Egger M, Burton A, Scoggins $C$, Ross $M$, Stromberg A et al. Prognostic implications of anatomic location of primary cutaneous melanoma of $1 \mathrm{~mm}$ or thicker. 2011;202(6):659-64; discussion 64-5. doi:10.1016/j.amjsurg.2011.06.048. 
5. Howard MD, Wee E, Wolfe R, McLean CA, Kelly JW, Pan Y. Anatomic location of primary melanoma: Survival differences and sun exposure. J Am Acad Dermatol. 2019;81(2):500-9. doi:10.1016/j.jaad.2019.04.034.

6. Garbe C, Büttner P, Bertz J, Burg G, d'Hoedt B, Drepper H et al. Primary cutaneous melanoma. Identification of prognostic groups and estimation of individual prognosis for 5093 patients. 1995;75(10):2484-91. doi:10.1002/1097-0142(19950515)75:10<2484::aid-cncr2820751014>3.0.co;2-u.

7. Gillgren P, Månsson-Brahme E, Frisell J, Johansson H, Larsson O, Ringborg UJAo. Epidemiological characteristics of cutaneous malignant melanoma of the head and neck-a population-based study. 1999;38(8):1069-74. doi:10.1080/028418699432383.

8. Lesage C, Barbe C, Le Clainche A, Lesage FX, Bernard P, Grange F. Sex-related location of head and neck melanoma strongly argues for a major role of sun exposure in cars and photoprotection by hair. J Invest Dermatol. 2013;133(5):1205-11. doi:10.1038/jid.2012.405.

9. Dabouz F, Barbe C, Lesage C, Le Clainche A, Arnoult G, Hibon E et al. Clinical and histological features of head and neck melanoma: a population-based study in France. Br J Dermatol. 2015;172(3):707-15. doi:10.1111/bjd.13489.

10. Juzeniene A, Micu E, Porojnicu A, Moan JJJotEAoD, JEADV V. Malignant melanomas on head/neck and foot: differences in time and latitudinal trends in Norway. 2012;26(7):821-7. doi:10.1111/j.14683083.2011.04162.x.

11. Ghiasvand R, Robsahm T, Green A, Rueegg C, Weiderpass E, Lund E et al. Association of Phenotypic Characteristics and UV Radiation Exposure With Risk of Melanoma on Different Body Sites. 2019;155(1):39-49. doi:10.1001/jamadermatol.2018.3964.

12. Tseng W, Martinez SJTJosr. Tumor location predicts survival in cutaneous head and neck melanoma. 2011;167(2):192-8. doi:10.1016/j.jss.2010.10.008.

13. de Giorgi V, Rossari S, Gori A, Grazzini M, Savarese I, Crocetti E et al. The prognostic impact of the anatomical sites in the 'head and neck melanoma': scalp versus face and neck. Melanoma Res. 2012;22(5):402-5. doi:10.1097/CMR.0b013e3283577b96.

14. Shi K, Camilon PR, Roberts JM, Meier JD. Survival Differences Between Pediatric Head and Neck Versus Body Melanoma in the Surveillance, Epidemiology, and End Results Program. Laryngoscope. 2020. doi:10.1002/lary.28711.

15. Helsing P, Robsahm TE, Vos L, Rizvi SM, Akslen LA, Veierod MB. Cutaneous head and neck melanoma (CHNM): A population-based study of the prognostic impact of tumor location. J Am Acad Dermatol. 2016;75(5):975-82 e2. doi:10.1016/j.jaad.2016.06.048.

16. Hoersch B, Leiter U, Garbe CJTBjod. Is head and neck melanoma a distinct entity? A clinical registrybased comparative study in 5702 patients with melanoma. 2006;155(4):771-7. doi:10.1111/j.13652133.2006.07455.x.

17. Ringborg U, Afzelius L, Lagerlöf B, Adami H, Augustsson I, Blomqvist E et al. Cutaneous malignant melanoma of the head and neck. Analysis of treatment results and prognostic factors in 581 patients: a report from the Swedish Melanoma Study Group. 1993;71(3):751-8. doi:10.1002/10970142(19930201)71:3<751::aid-cncr2820710317>3.0.co;2-9. 
18. Xie C, Pan Y, McLean C, Mar V, Wolfe R, Kelly J. Impact of scalp location on survival in head and neck melanoma: A retrospective cohort study. J Am Acad Dermatol. 2017;76(3):494-8 e2. doi:10.1016/j.jaad.2016.08.009.

19. Ali AN, Switchenko JM, Kim S, Kowalski J, El-Deiry MW, Beitler JJ. A model and nomogram to predict tumor site origin for squamous cell cancer confined to cervical lymph nodes. Cancer. 2014;120(22):346976. doi:10.1002/cncr.28901.

20. Zhang N, Zhang J, Zhang H, Liu Y, Zhao W, Wang L et al. Individualized Prediction of Survival Benefit from Postmastectomy Radiotherapy for Patients with Breast Cancer with One to Three Positive Axillary Lymph Nodes. Oncologist. 2019;24(12):e1286-e93. doi:10.1634/theoncologist.2019-0124.

21. Hanrahan EO, Gonzalez-Angulo AM, Giordano SH, Rouzier R, Broglio KR, Hortobagyi GN et al. Overall survival and cause-specific mortality of patients with stage T1a,bN0M0 breast carcinoma. J Clin Oncol. 2007;25(31):4952-60. doi:10.1200/JC0.2006.08.0499.

22. Albert JM, Liu DD, Shen Y, Pan IW, Shih YC, Hoffman KE et al. Nomogram to predict the benefit of radiation for older patients with breast cancer treated with conservative surgery. J Clin Oncol. 2012;30(23):2837-43. doi:10.1200/JC0.2011.41.0076.

23. Claeson M, Baade P, Brown S, Soyer HP, Smithers BM, Green AC et al. Clinicopathological factors associated with death from thin (</= $1.00 \mathrm{~mm}$ ) melanoma. Br J Dermatol. 2020;182(4):927-31. doi:10.1111/bjd.18560.

24. Shen W, Sakamoto N, Yang L. Melanoma-specific mortality and competing mortality in patients with non-metastatic malignant melanoma: a population-based analysis. BMC Cancer. 2016;16:413. doi:10.1186/s12885-016-2438-3.

25. Yang J, Pan Z, Zhao F, Feng X, Liu Q, Li Y et al. A nomogram for predicting survival in patients with nodular melanoma: A population-based study. 2019;98(24):e16059.

doi:10.1097/md.0000000000016059.

26. Xiao Y, Peng S, Hu Y, Zhang J, Cao X. Development and validation of prognostic nomogram in patients with nonmetastatic malignant melanoma: a SEER population-based study. Cancer Med. 2020;9(22):8562-70. doi:10.1002/cam4.3318.

27. Liu H, Xu YB, Guo CC, Li MX, Ji JL, Dong RR et al. Predictive value of a nomogram for melanomas with brain metastases at initial diagnosis. Cancer Med. 2019;8(18):7577-85. doi:10.1002/cam4.2644.

28. MacKie R, Bray C, Vestey J, Doherty V, Evans A, Thomson D et al. Melanoma incidence and mortality in Scotland 1979-2003. 2007;96(11):1772-7. doi:10.1038/sj.bjc.6603801.

29. Ciocan D, Barbe C, Aubin F, Granel-Brocard F, Lipsker D, Velten M et al. Distinctive features of melanoma and its management in elderly patients: a population-based study in France. 2013;149(10):1150-7. doi:10.1001/jamadermatol.2013.706.

30. Stanienda-Sokół K, Salwowska N, Sławińska M, Wicherska-Pawłowska K, Lorenc A, Wcisło-Dziadecka D et al. Primary Locations of Malignant Melanoma Lesions Depending on Patients' Gender and Age. 2017;18(11):3081-6. doi:10.22034/apjcp.2017.18.11.3081.

31. Gillgren P, Månsson-Brahme E, Frisell J, Johansson H, Larsson O, Ringborg UJTL. A prospective population-based study of cutaneous malignant melanoma of the head and neck. 2000;110(9):1498- 
504. doi:10.1097/00005537-200009000-00017.

32. Lachiewicz A, Berwick M, Wiggins C, Thomas NJAod. Survival differences between patients with scalp or neck melanoma and those with melanoma of other sites in the Surveillance, Epidemiology, and End Results (SEER) program. 2008;144(4):515-21. doi:10.1001/archderm.144.4.515.

33. Wood RP, Heyworth JS, McCarthy NS, Mauguen A, Berwick M, Thomas NE et al. Association of Known Melanoma Risk Factors with Primary Melanoma of the Scalp and Neck. Cancer Epidemiol Biomarkers Prev. 2020;29(11):2203-10. doi:10.1158/1055-9965.EPI-20-0595.

34. Volkovova K, Bilanicova D, Bartonova A, Letašiová S, Dusinska MJEhagass. Associations between environmental factors and incidence of cutaneous melanoma. Review. 2012:S12. doi:10.1186/1476069x-11-s1-s12.

35. Dessinioti C, Dimou N, Geller A, Stergiopoulou A, Lo S, Keim U et al. Distinct Clinicopathological and Prognostic Features of Thin Nodular Primary Melanomas: An International Study from 17 Centers. 2019;111(12):1314-22. doi:10.1093/jnci/djz034.

36. Dessinioti C, Geller A, Stergiopoulou A, Swetter S, Baltas E, Mayer J et al. Association of Skin Examination Behaviors and Thinner Nodular vs Superficial Spreading Melanoma at Diagnosis. 2018;154(5):544-53. doi:10.1001/jamadermatol.2018.0288.

37. Saaiq M, Zalaudek I, Rao B, Lee Y, Rudnicka L, Czuwara J et al. A brief synopsis on scalp melanoma. Dermatol Ther. 2020;33(4):e13795. doi:10.1111/dth.13795.

\section{Table 1}

Table 1 Comparison of head and neck melanoma (HNM) with body melanoma (BM)

\begin{tabular}{|c|c|c|c|}
\hline & HNM & p value & \\
\hline Total & $15071(21)^{*}$ & $55534(79)^{* *}$ & \\
\hline Age, mean/median (y) & $64.6 / 67$ & $57.2 / 58$ & \\
\hline Age (n \%) & & & $<0.001$ \\
\hline$<=58$ & $4816(32.0)$ & $28941(52.1)$ & \\
\hline $59-78$ & $6908(45.8)$ & $21114(38.0)$ & \\
\hline$>=79$ & $3347(22.2)$ & $5479(9.9)$ & \\
\hline Sex (n \%) & & & $<0.001$ \\
\hline Male & $10913(72.4)$ & 29066 (52.3) & \\
\hline Female & $4158(27.6)$ & $26468(47.7)$ & \\
\hline Race (n \%) & & & $<0.001$ \\
\hline White & $14928(99.1)$ & $54684(98.5)$ & \\
\hline${ }^{\mathrm{a}}$ Others & $143(0.9)$ & $850(1.5)$ & \\
\hline Histology (n \%) & & & $<0.001$ \\
\hline NM & $2352(15.6)$ & $7905(14.2)$ & \\
\hline LMM & $4798(31.8)$ & $3638(6.6)$ & \\
\hline SMM & $6168(40.9)$ & $38748(69.8)$ & \\
\hline${ }^{\mathbf{b}}$ Other Melanoma & $1753(11.6)$ & $5243(9.4)$ & \\
\hline Thickness (n \%) & & & $<0.001$ \\
\hline$<0.6 \mathrm{~mm}$ & $6717(44.6)$ & $25301(45.6)$ & \\
\hline $0.6-1.0 \mathrm{~mm}$ & $2601(17.3)$ & $11998(21.6)$ & \\
\hline $1.01-2.0 \mathrm{~mm}$ & $2394(15.9)$ & $8746(15.7)$ & \\
\hline $2.01-4.0 \mathrm{~mm}$ & 1759 (11.7) & $5337(9.6)$ & \\
\hline$>4.0 \mathrm{~mm}$ & $1600(10.6)$ & $4152(7.5)$ & \\
\hline Ulceration (n \%) & & & 0.004 \\
\hline No & 12651 (83.9) & $47153(84.9)$ & \\
\hline Yes & $2420(16.1)$ & $8381(15.1)$ & \\
\hline
\end{tabular}


Stage (n \%)

Localized

Regional

Distant

Metastases (n \%)

No

Yes

Surgery (n \%)

No

Yes

Radiation (n \%)

No/Unknown

Yes

Chemotherapy (n \%)

No/Unknown

Yes
$12944(85.9)$

1868 (12.4)

259 (1.7)

14861 (98.6)

$210(1.4)$

$342(2.3)$

$14729(97.7)$

14519 (96.3)

552 (3.7)

14869 (98.7)

$202(1.3)$
$<0.001$

49320 (88.8)

5408 (9.7)

806 (1.5)

$<0.001$

54980 (99.0)

554 (1.0)

$1092(2.0)$

$54442(98.0)$

55055 (99.1)

$479(0.9)$

54833 (98.7)

701 (1.3)

NM, nodular melanoma; LMM, Lentigo maligna melanoma; SSM, superficial spreading melanoma.

*HNM constitute $21 \%$ of all cutaneous melanomas.

**BM constitute $79 \%$ of all cutaneous melanomas.

a Others=black, Asian or pacific islander, American indian/Alaska native.

b Other melanoma: International Classification of Diseases for Oncology-O-3, codes 8722-8723, 8730, 8740-8741, 8744-8746, 8761, 8770-8774, 8780.

\section{Figures}

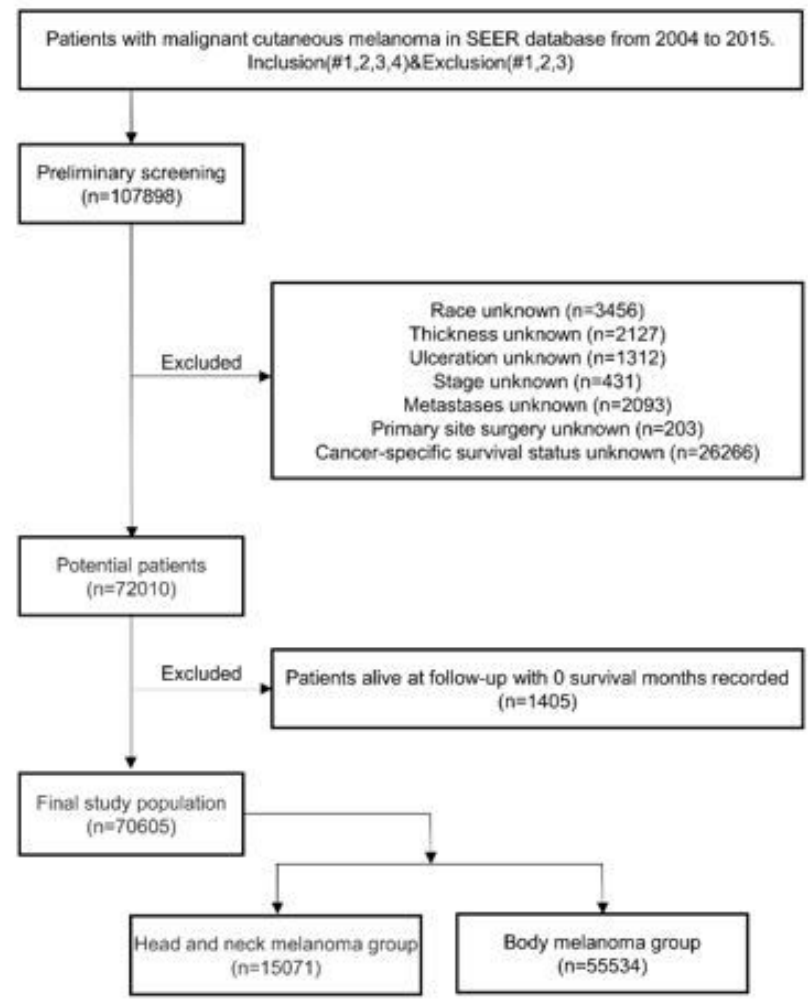

a
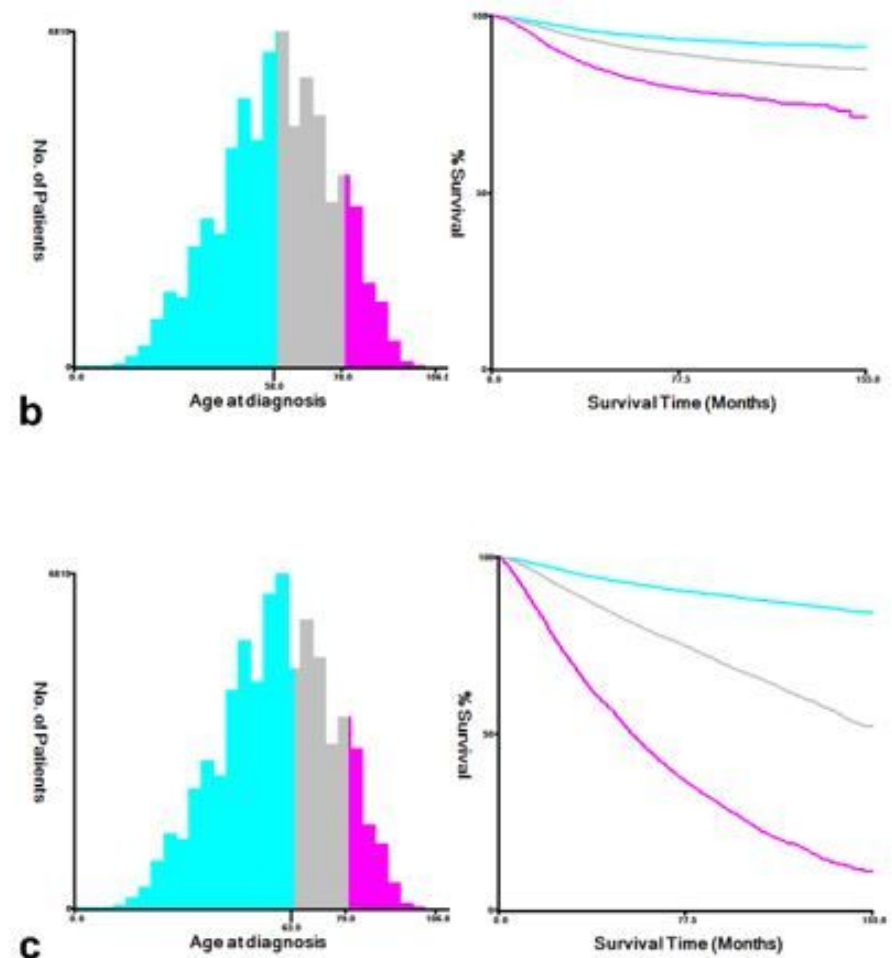


\section{Figure 1}

(a) Flowchart of patient selection. (b) The optimal cut-off values for age based on cancer-specific survival were $<=58,59-78$, and $>=79$ years old. (c) The optimal cut-off values for age based on overall survival were $<=63,64-79$, and $>=80$ years old.
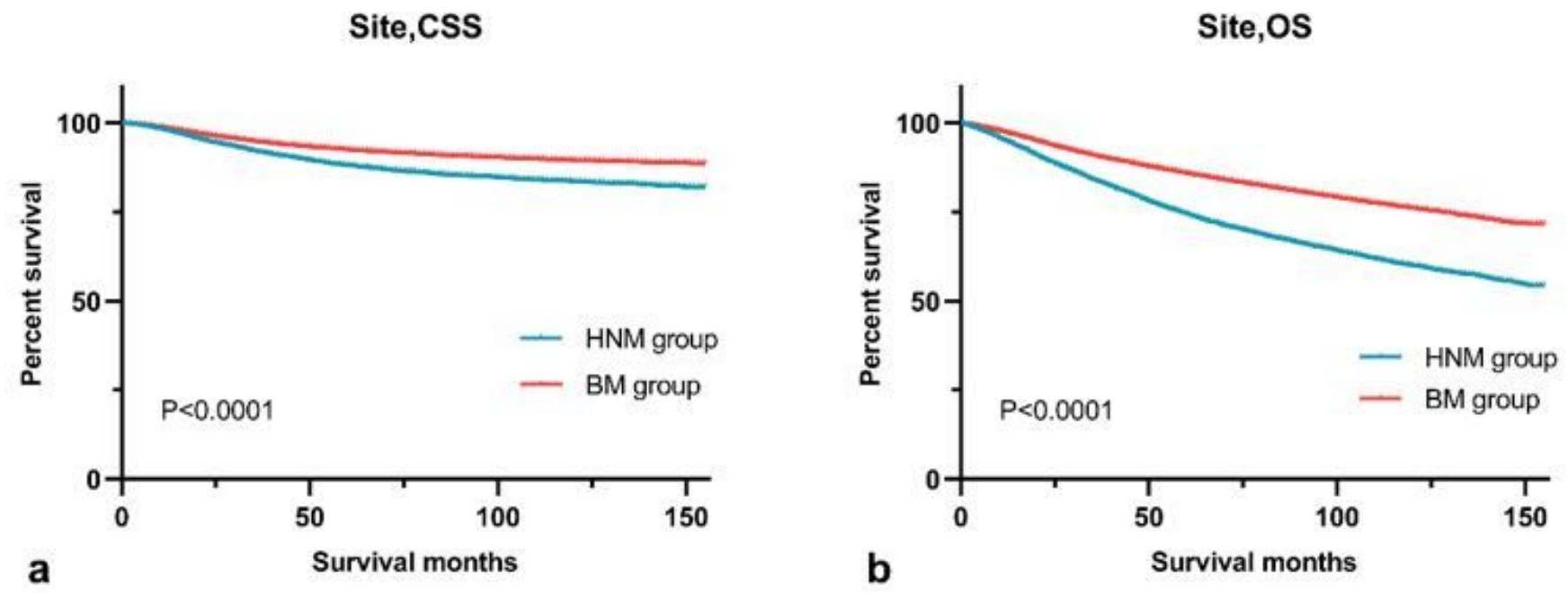

Figure 2

Kaplan-Meier curves of cancer-specific and overall survival based on anatomic site. (a) cancer-specific survival (CSS); (b) overall survival (OS). HNM = head and neck melanoma; BM = body melanoma. 

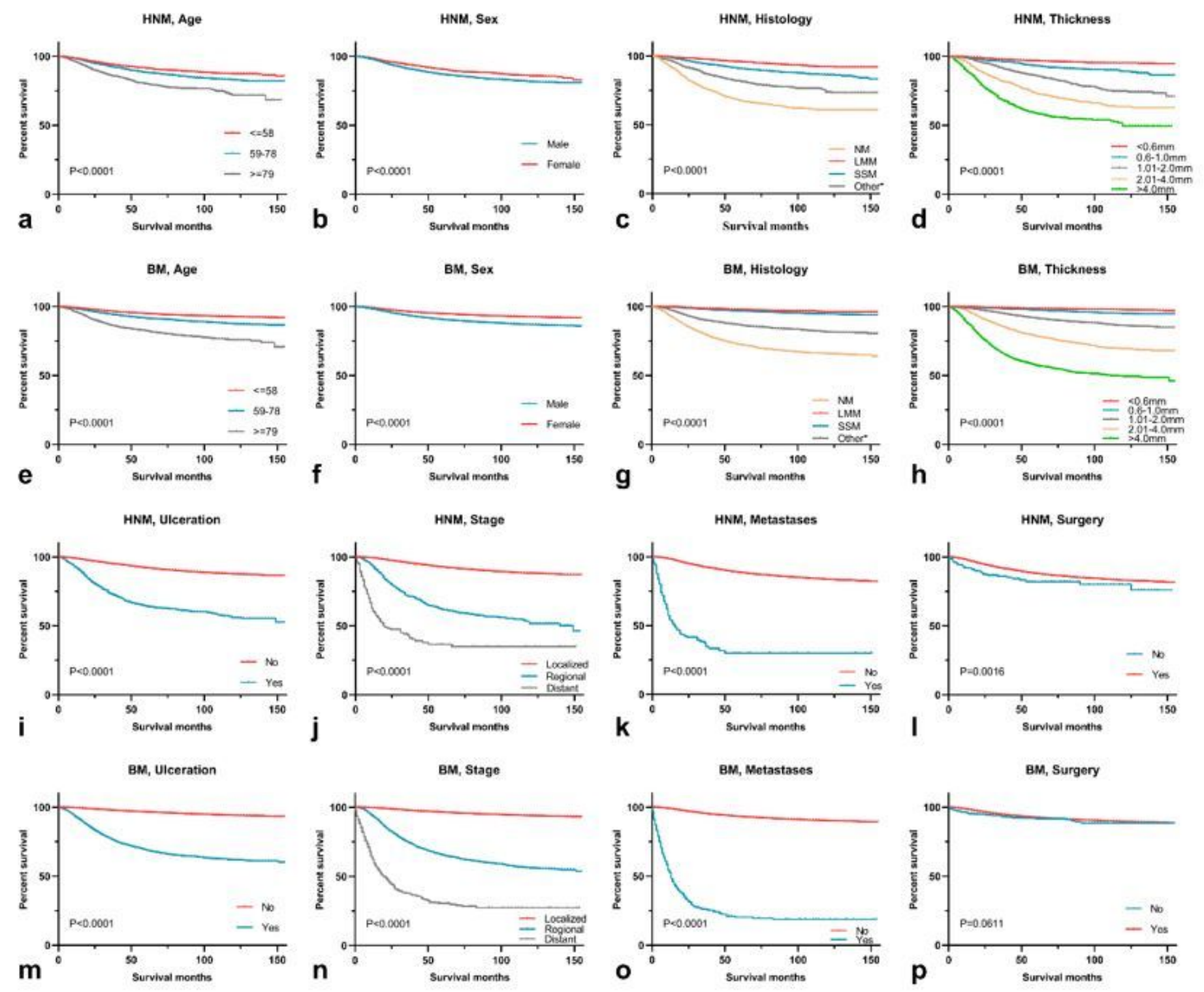

\section{Figure 3}

Kaplan-Meier curves of CSS were delineated based on the identified independent prognostic factors, including age, sex, histology, thickness, ulceration, stage, metastases, and surgery (a-d, i-I) for HNM training cohort, (e-h, m-p) for BM training cohort. CSS = cancer-specific survival; $\mathrm{HNM}=$ head and neck melanoma; $\mathrm{BM}=$ body melanoma 

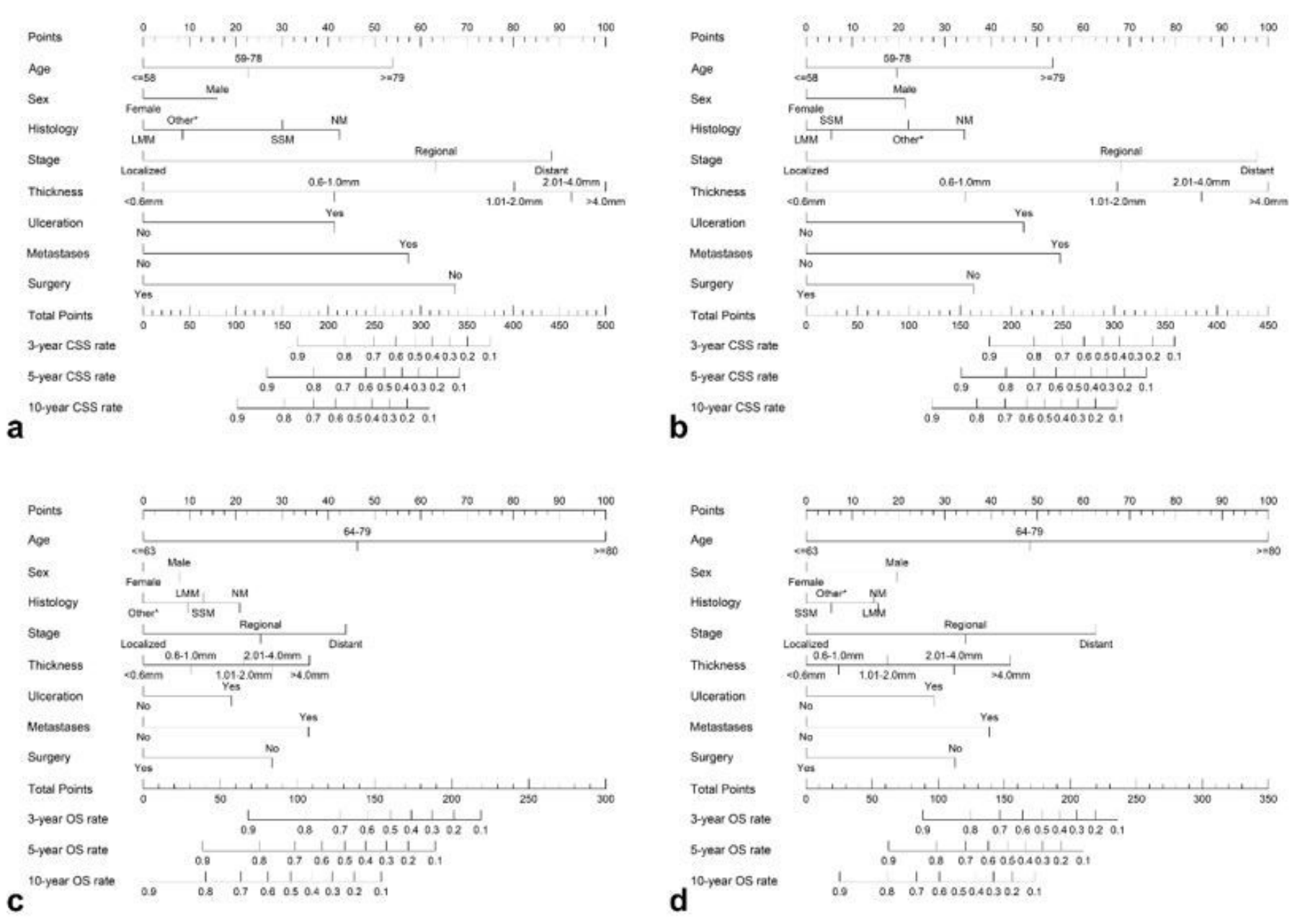

\section{Figure 4}

Nomograms predicted the individual 3-, 5- and 10-year survival rates of patients. $(\mathrm{a}, \mathrm{c})$ predicting CSS and OS for HNM training cohort; $(b, d)$ predicting CSS and OS for BM training cohort. CSS = cancer-specific survival; $\mathrm{OS}$ = overall survival; $\mathrm{HNM}=$ head and neck melanoma; $\mathrm{BM}=$ body melanoma. 

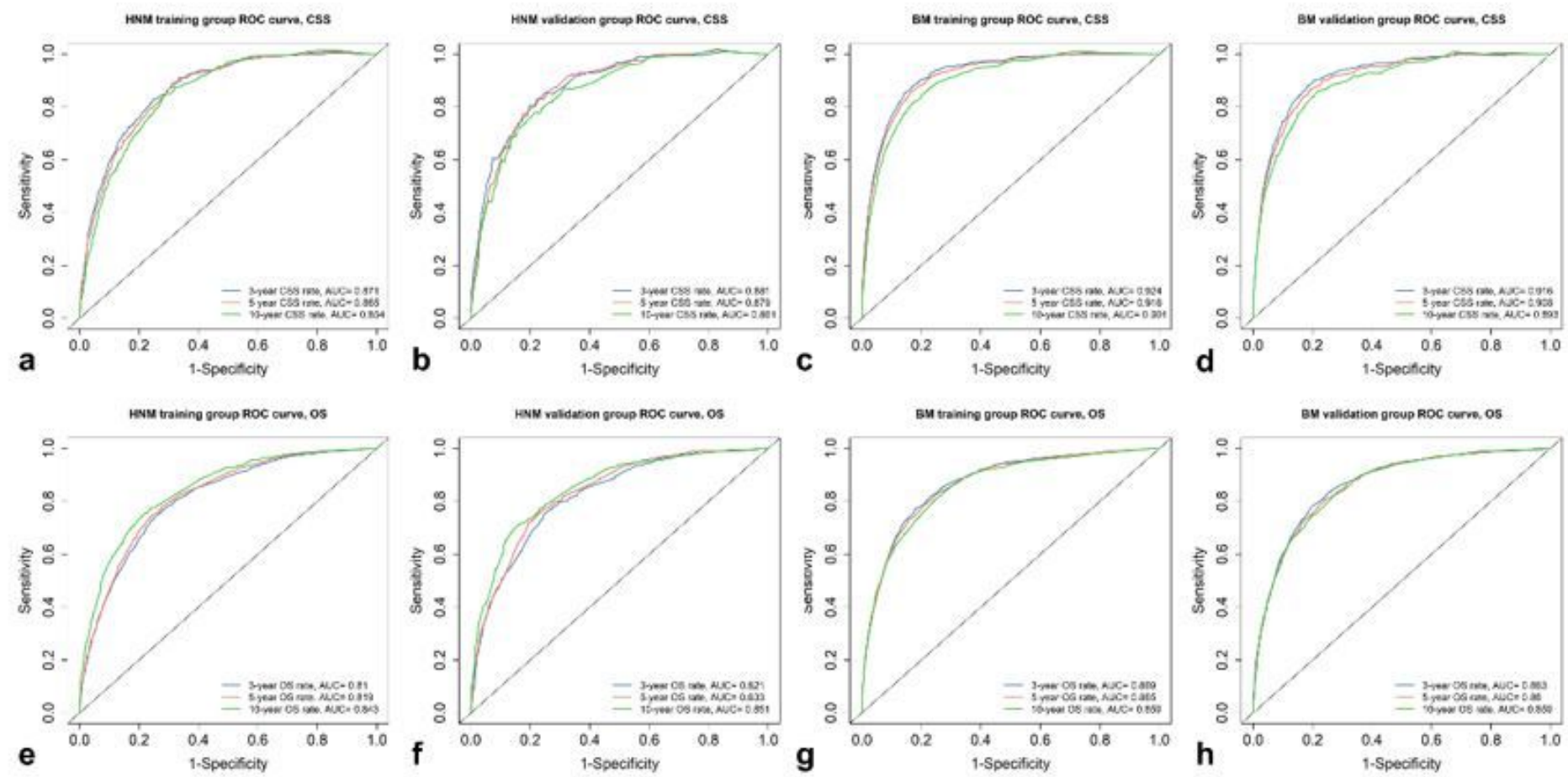

\section{Figure 5}

ROC curves evaluated the predictive ability of the nomograms. a-d predicted 3-, 5- and 10-year CSS rates for HNM training and validation cohort, BM training and validation cohort, respectively. e-h predicted 3-, 5- and 10-year OS rates for HNM training and validation cohort, BM training and validation cohort, respectively. 

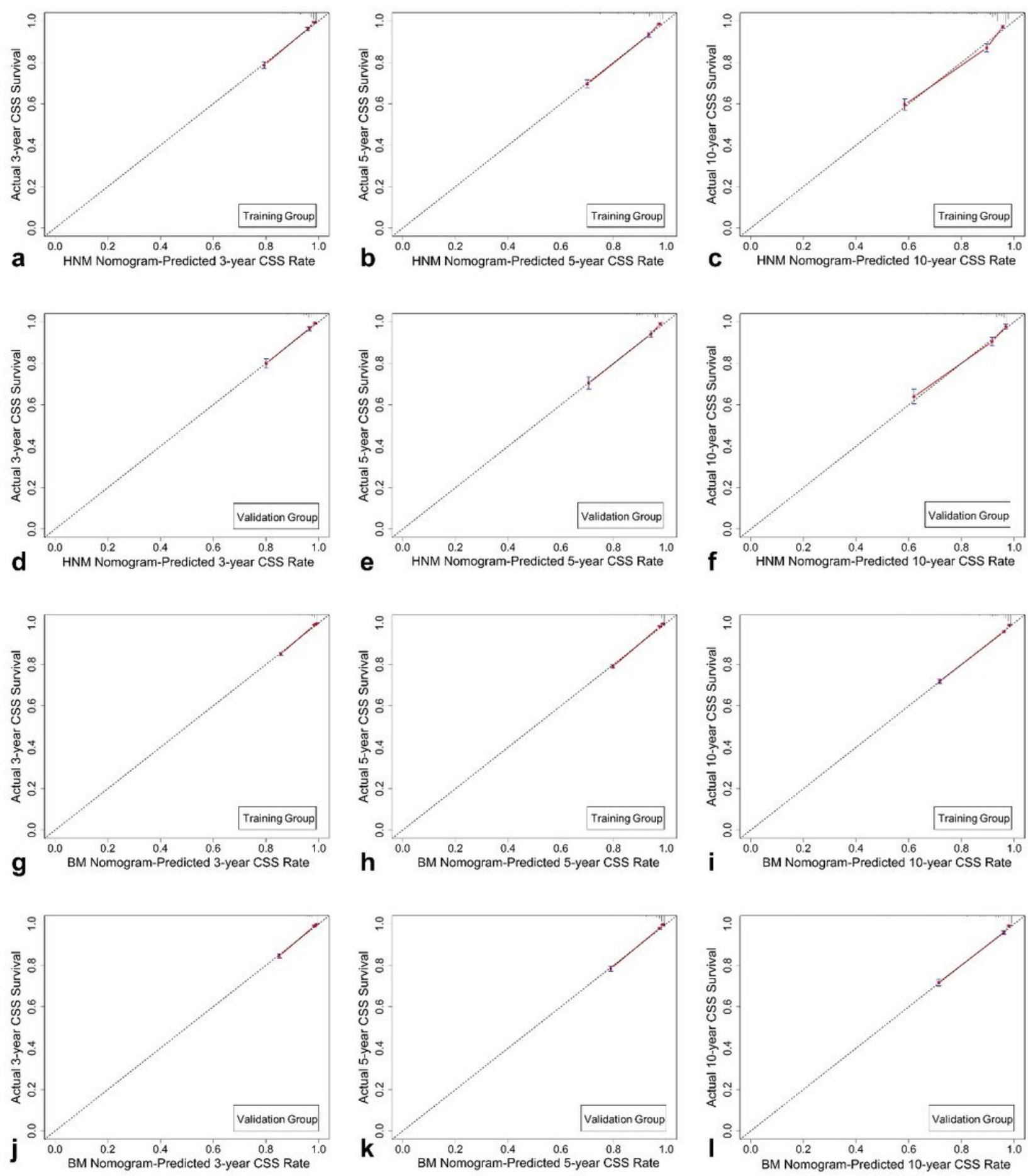

Figure 6

Calibration curves showed the probability of 3-, 5- and 10-year cancer-specific survival (CSS) between the nomogram prediction and the actual observation. Perfect prediction would correspond to the 45-degree line. The calibration curves predicted CSS of patients with HNM in the training cohort $(\mathrm{a}-\mathrm{c})$ and in the validation cohort (d-f). The calibration curves predicted CSS of patients with BM in the training cohort ( $g-h)$ and in the validation cohort (j-l). 


\section{Supplementary Files}

This is a list of supplementary files associated with this preprint. Click to download.

- SupplementaryMaterial.docx 